\title{
Commentary: X-ray vision, a superpower against postoperative pain?
}

\author{
John P. Scott, MD
}

In the current issue of the Journal, Schmehil and colleagues ${ }^{1}$ report the results of a single-center retrospective review of fluoroscopically guided thoracic epidural (fTEA) analgesia in patients undergoing congenital cardiac surgery. The primary aim was to demonstrate successful thoracic epidural catheter placement using fluoroscopy. The authors postulate that incorporating fTEA confirmed with an epidurogram after placement improves safety and efficacy. This is supported by the fact that 119 of 120 patients underwent successful placement without complications. Additional outcome measures reported included duration of intubation and hospital length of stay, but no comparative data or pain related measures of efficacy were included.

Neuraxial and regional anesthesia have been identified as key components of Enhanced Recovery After Surgery (ERAS) protocols, including following cardiac surgery. These adjuvants are purported to reduce opioid requirements, leading to improved outcomes and resource use. Epidural and paravertebral blocks have been most frequently studied; however, emerging non-neuraxial truncal regional anesthetic techniques, including pectointercostal fascial blocks and erector spinae plane blocks, may offer similar analgesic benefits with reduced complication potential, especially in the setting of systemic anticoagulation. ${ }^{2-6}$

\footnotetext{
From the Sections of Pediatric Anesthesiology and Pediatric Critical Care, Departments of Anesthesiology and Pediatrics, Medical College of Wisconsin, Milwaukee, Wis.

Disclosures: The author reported no conflicts of interest.

The Journal policy requires editors and reviewers to disclose conflicts of interest and to decline handling or reviewing manuscripts for which they may have a conflict of interest. The editors and reviewers of this article have no conflicts of interest.

Received for publication Nov 3, 2021; revisions received Nov 3, 2021; accepted for publication Nov 5, 2021; available ahead of print Nov 11, 2021.

Address for reprints: John P. Scott, MD, Departments of Anesthesiology and Pediatrics, Medical College of Wisconsin, 9000 W Wisconsin Ave, Milwaukee, WI 53122 (E-mail: scottjake@mcw.edu).

JTCVS Techniques 2022;11:67-8

2666-2507

Copyright (c) 2021 The Author(s). Published by Elsevier Inc. on behalf of The American Association for Thoracic Surgery. This is an open access article under the CC BY-NC-ND license (http://creativecommons.org/licenses/by-nc-nd/4.0/).

https://doi.org/10.1016/j.xjtc.2021.11.009
}

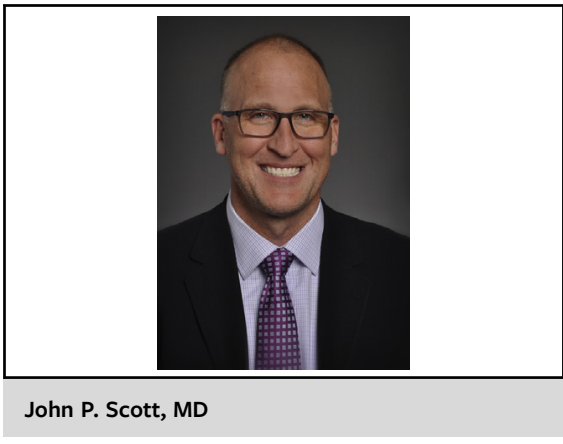

CENTRAL MESSAGE

Fluoroscopic confirmation of

proper thoracic epidural place-

ment may augment the safety

and utility of this regional anes-

thetic technique in congenital

cardiac surgery.

This reduced risk profile underscores the need to demonstrate a benefit to patients rather than merely reporting the absence of adverse of events.

To truly evaluate the efficacy of fTEA in this setting, comparative studies incorporating pain-associated outcome measures are required. It is noteworthy that analyses of regional versus general anesthesia in adults and children have yielded conflicting data, with many newer studies failing to demonstrate an outcome benefit. ${ }^{7,8}$ This highlights that ultimately the type of anesthetic technique employed may be less important than making sure that it is done well, as Schmehil and colleagues have done in this study.

\section{References}

1. Schmehil C, Lee K-J, Casella S, Millan D. Thoracic epidural anesthesia in congenital heart surgery. J Thorac Cardiovasc Surg Tech. 2022;11:64-6.

2. Roy N, Brown ML, Parra MF, Sleeper LA, Alrayashi W, Nasr VG, et al. Bilateral erector spinae blocks decrease perioperative opioid use after pediatric cardiac surgery. J Cardiothorac Vasc Anesth. 2021;35:2082-7.

3. Kaushal B, Chauhan S, Magoon R, Krishna NS, Saini K, Bhoi D, et al. Efficacy of bilateral erector spinae plane block in management of acute postoperative surgical pain after pediatric cardiac surgeries through a midline sternotomy. J Cardiothorac Vasc Anesth. 2020;34:981-6.

4. Voulgarelis S, Halenda GM, Tanem JM. A novel use of liposomal bupivacaine in erector spinae plane block for pediatric congenital cardiac surgery. Case Rep Anesthesiol. 2021;2021:5521136.

5. Holland EL, Bosenberg AT. Early experience with erector spinae plane blocks in children. Paediatr Anaesth. 2020;30:96-107. 
6. Sahajanandan R, Varsha AV, Kumar DS, Kuppusamy B, Karuppiah S, Shukla V, et al. Efficacy of paravertebral block in "Fast-tracking" pediatric cardiac surgery-experiences from a tertiary care center. Ann Card Anaesth. 2021;24:24-9. 7. McCann ME, de Graaff JC, Dorris L, Disma N, Withington D, Bell G, et al Neurodevelopmental outcome at 5 years of age after general anaesthesia or awake-regional anaesthesia in infancy (GAS): an international, multicentre, randomised, controlled equivalence trial. Lancet. 2019;393:664-77.

8. Neuman MD, Feng R, Carson JL, Gaskins LJ, Dillane D, Sessler DI, et al. Spinal anesthesia or general anesthesia for hip surgery in older adults. $N$ Engl J Med. 2021;385:2025-35. 